\title{
A Third Order Sigma-Delta Modulator
}

\author{
D. Milovanović, M. Savić and M. Nikolić
}

Abstract - The design of a sigma-delta modulator is presented in this paper. Third order mash structure is chosen and implemented. Top-down methodology is used and described. Design fulfills imposed requirements which is verified with postlayout transistor level simulation results.

\section{INTRODUCTION}

The use of oversampling sigma-delta modulators in the integration of high-resolution analog-to-digital converters has shown promise for overcoming the analog component limitations inherent in modern VLSI technologies. Sigma-delta modulators employ coarse quantization enclosed in one or more feedback loops. By sampling at a frequency that is much greater than the signal bandwidth, it is possible for the feedback loops to shape the quantization noise so that most of the noise power is shifted out of the signal band. The out of band noise can then be attenuated with a digital filter. The degree to which the quantization noise can be attenuated depends on the order of the noise shaping and the oversampling ratio.

As a part of wider project sigma-delta modulator was designed. It represents an $\mathrm{A} / \mathrm{D}$ part of a power meter IC. Requirements imposed were: SNDR and dynamic range $>$ $90 \mathrm{~dB}$ for maximum input swing of $125 \mathrm{mV}$ differential at $50 \mathrm{~Hz}$. Oversampling ratio is 128 with clock frequency of $524288 \mathrm{~Hz}$ which gives bandwidth of $2048 \mathrm{~Hz}$. To fulfill the requirements we chose the third-order modulator. In theory the second-order modulator is sufficient [1], but the third-order will give enough margins for circuit nonidealities and process variations. The modulator comprises a cascade of a second-order stage, hereafter referred as a 21 architecture. A cascaded architecture was adopted primarily for two reasons. First, cascading first and secondorder modulators eliminates the stability problems associated with third and higher order single stage modulators while retaining essentially the same quantization noise performance [2]. Second, any baseband noise tones produced in the second-order stage are largely suppressed by the succeeding first-order, and the remaining quantization noise is nearly white. The $2-1$ architecture is preferable to alternative cascaded third-order architectures because it is less sensitive to component mismatch.

This paper is divided into following parts. Section II and III describes implementation of 2-1 architecture. In section IV behavioral simulation is done to verify

D. Milovanović, M. Savić and M. Nikolić are with the Department of Electronics, Faculty of Electronic Engineering, University of Niš, Beogradska 14, 18000 Niš, Serbia \& Montenegro, E-mail: msavic@elfak.ni.ac.yu architecture implementation. Finally, in section V design of an experimental third-order modulator are given.

\section{MODULATOR DESIGN}

The 2-1 architecture is implemented by combining three summing integrators with two comparators and two 1-b D/A converters, as shown in Fig. 1 [3]. The most important building block in this architecture is a summing integrator, for which the output $w$ is the delayed integration of a weighted sum of inputs $v_{m}$. In the time domain, the output is

$$
w((n+1) T s)=w(n T s)+\sum_{m} a_{m} v_{m}(n T s)
$$

where $T s$ is the modulator's sampling period. The $z$ transform of the output is

$$
W(z)=\frac{z^{-1}}{1-z^{-1}} \sum_{m} a_{m} V_{m}(z)
$$

where $V_{m}(z)$ is the $z$ transform of $v_{m}(n T s)$

The remaining building blocks in the analog portion of the modulator are comparators and 1-b D/A converters. The comparator circuits acts as a 1-b A/D converters that map their inputs into one of two digital output codes. The two digital output codes are then mapped back into analog levels by the $\mathrm{D} / \mathrm{A}$ converters. If the two output codes of the comparators are defined as $\pm 1 / 2$, then the $\mathrm{D} / \mathrm{A}$ converters, neglecting $\mathrm{D} / \mathrm{A}$ errors, can be represented simply by gain block.

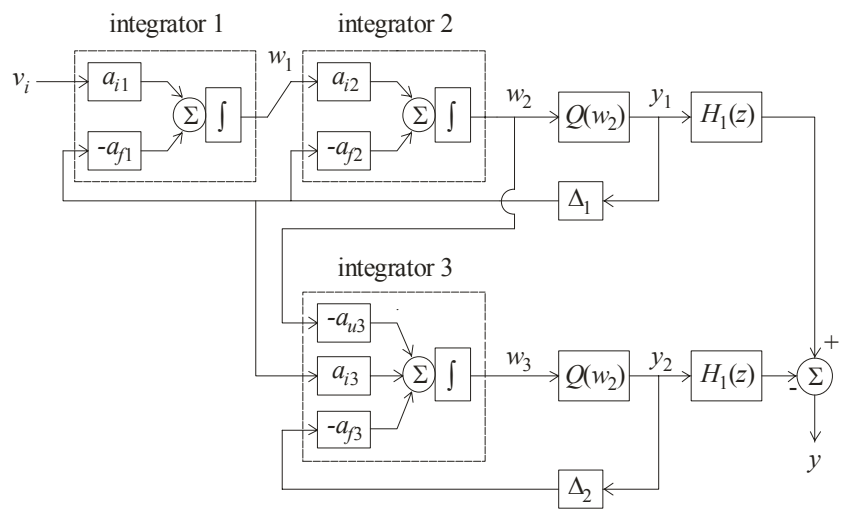

Fig. 1. 2-1 architecture implementation 
Digital part of modulator consist of error cancellation filters $H_{1}(z)$ and $H_{2}(z)$, which eliminates error from first stage of modulator. In $z$ domain transfer function of $H_{l}(z)$ and $H_{2}(z)$ are [3]

$$
\begin{aligned}
& H_{1}(z)=\frac{1}{2} z^{-1}\left(1+z^{-1}\right) \\
& H_{2}(z)=2\left(1-z^{-1}\right)^{2}
\end{aligned}
$$

The integrators' gains used in this design are summarized in Table I [3]

TABLE I

INTEGRATOR GAIN VALUES

\begin{tabular}{|l|l|l|l|l|l|l|l|}
\hline Gain & $\mathrm{a}_{\mathrm{i} 1}$ & $\mathrm{a}_{\mathrm{f} 1}$ & $\mathrm{a}_{\mathrm{i} 2}$ & $\mathrm{a}_{\mathrm{f} 2}$ & $\mathrm{a}_{\mathrm{u} 3}$ & $\mathrm{a}_{\mathrm{i} 3}$ & $\mathrm{a}_{\mathrm{f} 3}$ \\
\hline Value & 1.0 & 0.2 & 0.5 & 0.25 & 0.5 & 0.1 & 0.1 \\
\hline
\end{tabular}

\section{THE INTEGRATOR ARCHITECTURE}

Switched-capacitor integrators are used in this design. Two main sources of noise in switched-capacitor integrator are: thermal noise from the amplifier and equivalent $k T / C$ noise resulting from the integrator switches, and flicker noise from the amplifier. The thermal noise is limited by using sufficiently large capacitors to restrict the noise bandwidth. Minimum value used for capacitors in first integrator is $5 \mathrm{pF}\left(C_{R E F}\right)$. The flicker noise is attenuated using the correlated double sampling topology shown in Fig. 2 [4].

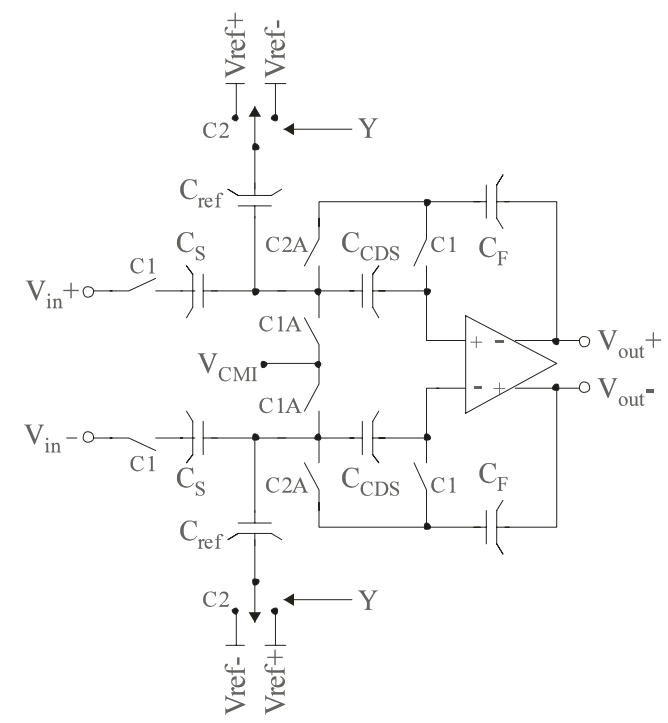

Fig. 2. Correlated double sampling integrator

In the double sampling integrator, a nonoverlapping two-phase clock is used. Switches $c_{1}$ and $c_{1 A}$ conduct during first clock phase, and switches $c_{2}$ and $c_{2 A}$ conduct during the second clock phase. Switches $c_{1 A}$ and $c_{2 A}$ are opened slightly ahead of switches $c_{1}$ and $c_{2}$ respectively to reduce signal-dependant charge injection onto sampling capacitors $C_{S}$ [5]. During the first phase, the input $V_{I}$ is sampled across $C_{S}$ and amplifier offset is sampled on $C_{C D S}$. In the second clock phase a charge proportional to the input voltage $V_{I}$ minus feedback voltage $V_{\text {ref }}$ is transferred from $C_{S}$ and $C_{r e f}$ to $C_{F}$, while dc offset and flicker noise of the amplifier are cancelled by the voltage stored on $C_{C D S}$. For proper operation, $C_{C D S}$ must be much larger then input capacitance of the amplifier. In this design $C_{C D S}$ is chosen to be $5 \mathrm{pF}$.

\section{BEHAVIORAL SIMULATION}

The analog circuit block cannot precisely perform their ideal function, so most of modulator nonidealities must be taken into account. Such are sampling jitter, $k T / C$ noise, and operational amplifier parameters (white noise, finite dc gain, finite bandwidth, slew rate and saturation voltages). Only the first integrator needs to be simulated with nonidealities, since their effects are not attenuated by noise shaping. For architecture implementation in Fig. 1 a simulation in Matlab Simulink environment was performed. Ideal modulator output spectrum for a $50 \mathrm{~Hz}$ $125 \mathrm{mV}$ sinusoidal input signal is shown in Fig. 3.

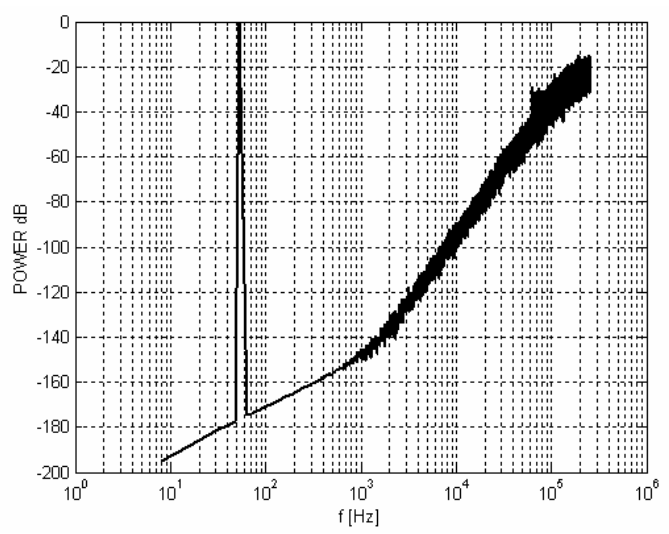

Fig. 3. Ideal modulator's output spectrum

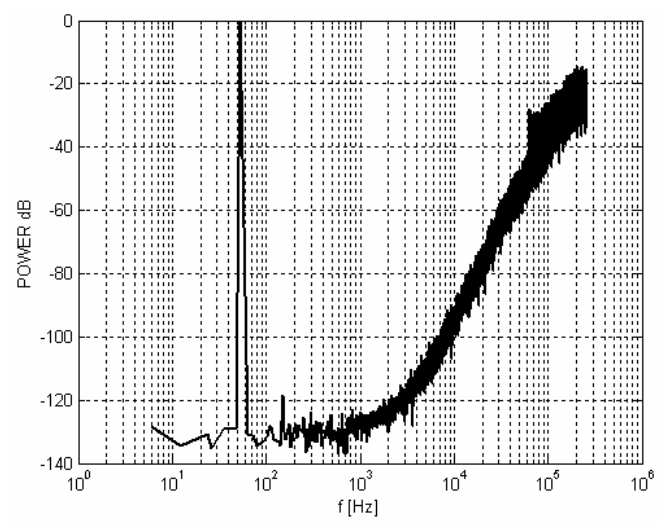

Fig. 5. Output spectrum with nonidealities 
Simulink model used to simulate nonidealities is shown in Fig. 4 [6]. Table II gives modulator parameters and values used in simulation. Only white noise is considered, while flicker noise and dc offset are neglected, because first integrator has correlated double sampling. Output spectrum for the same signal of the modulator with modeled nonidealities is shown in Fig. 5.

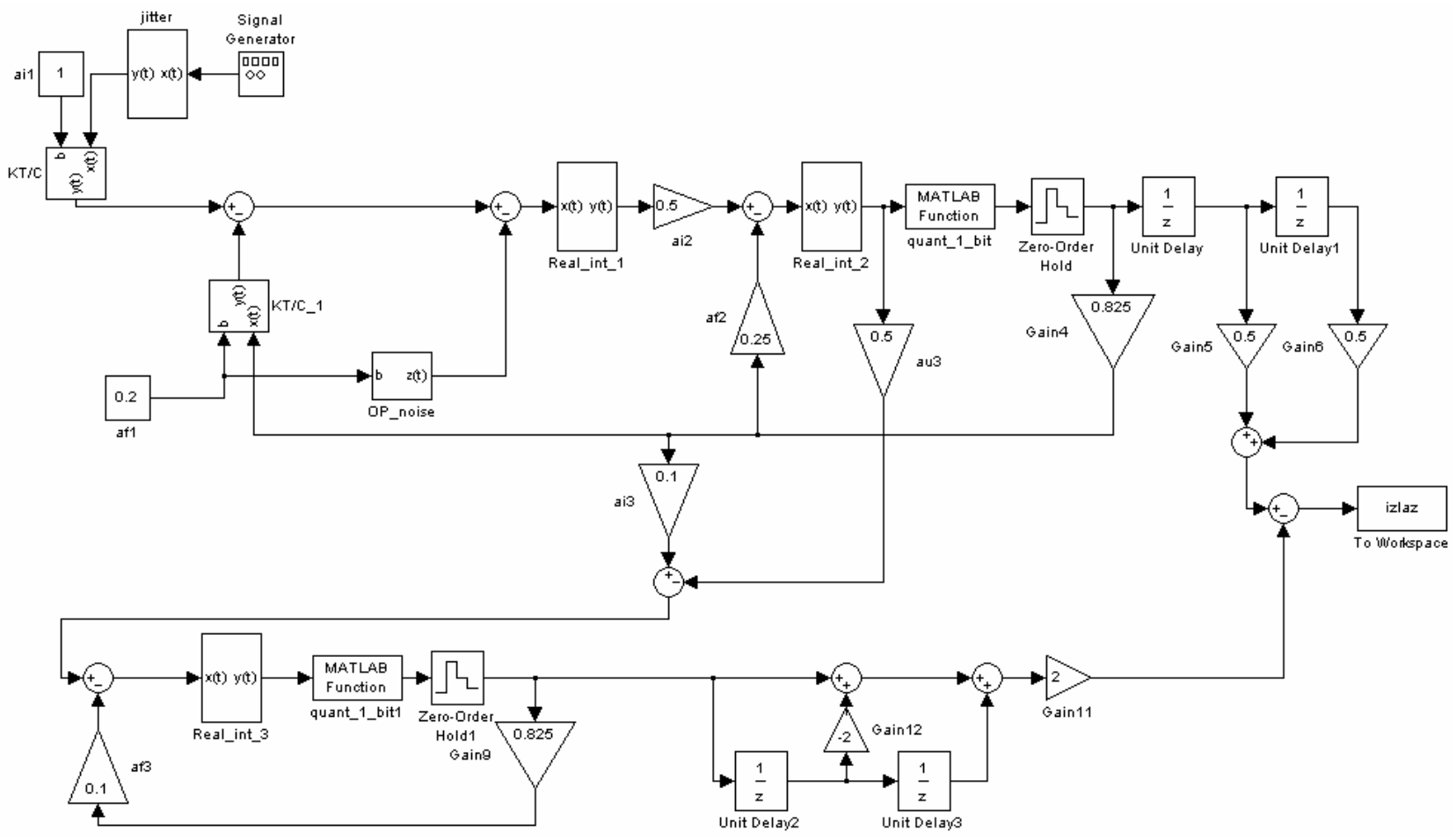

Fig. 4. Simulink model

TABLE II

MODULATOR NONIDEALITIES

\begin{tabular}{|l|l|}
\hline Modulator parameter & Value \\
\hline Sampling jitter & $20 \mathrm{~ns}$ \\
\hline Switches (kT/C $\mathrm{C}_{\mathrm{S}}$ ) noise & $25 \mathrm{pF}$ \\
\hline $\begin{array}{l}\text { Input-referred operational amplifier } \\
\text { noise (thermal) }\end{array}$ & $70 \mu \mathrm{V}_{\mathrm{rms}}$ \\
\hline Finite dc gain & $10^{3}$ \\
\hline Finite bandwidth & $2.5 \mathrm{MHz}$ \\
\hline Slew-rate & $4 \mathrm{~V} / \mu \mathrm{s}$ \\
\hline
\end{tabular}

\section{CIRCUIT DESIGN}

After behavioral level simulations we had enough parameters for transistor level implementation. All required analog blocks (operational amplifiers, bandgap reference, switches, capacitors and quantizer) were designed, simulated, and then layout is carried out.

The sigma-delta modulator depicted in Fig. 1 was designed for fabrication in $0.35-\mu \mathrm{m}$ CMOS technology. The operational amplifier used in integrators is the most critical element of the modulator. Behavioral simulation with nonidealities indicates that a slew rate of $4 \mathrm{~V} / \mu \mathrm{s}$, bandwidth of $2.5 \mathrm{MHz}$ is sufficient to meet performance objectives. Since the comparator can be designed to be quite fast, the settling speed of the integrator ultimately limits the achievable sampling rate of the modulator, even if complete settling is not required. The need for high speed, coupled with a relatively modest gain requirement of $60 \mathrm{~dB}$ to suppress harmonic distortion, encouraged the use of a single-stage amplifier [7].

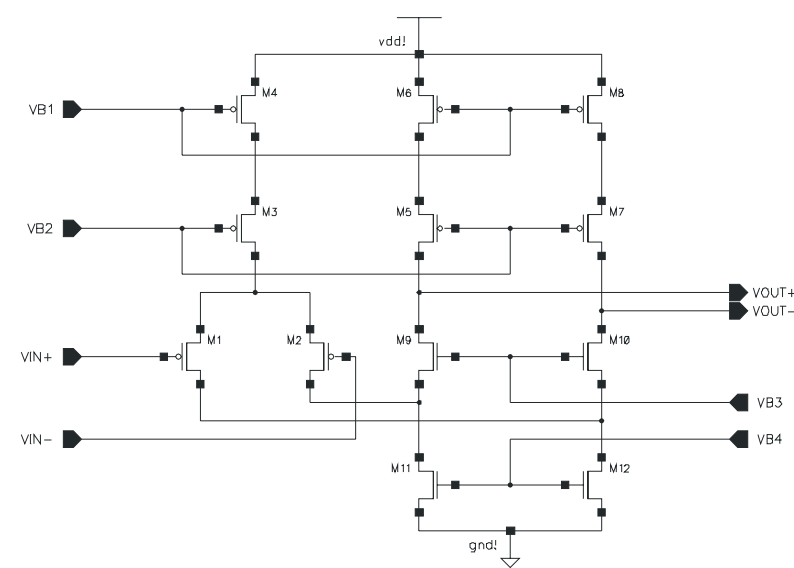

Fig. 6. Folded cascode op-amp

Figure 6 shows fully differential folded-cascoded operational amplifier used in design. The common-mode 
levels in the fully differentially amplifier are set by the common-mode feedback circuit shown in Fig. 7. Bias voltages are provided by a wide-swing cascode current mirror bias circuit.

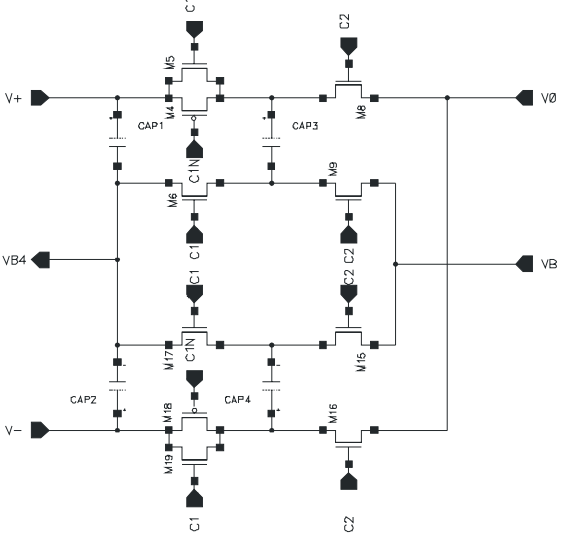

Fig. 7 CMFB circuit

Output spectrum obtained from transistor level simulations data for $8 \mathrm{kHz} 125 \mathrm{mV}$ sinusoidal input signal is shown in Fig. 8. Input signal frequency is $8 \mathrm{kHz}$ which is enough to have a reasonable simulation time, while giving enough samples (16 k samples) to perform a FFT. In Fig. 9 is shown layout of the modulator. Modulator occupies 0.66 $\mathrm{mm}^{2}$

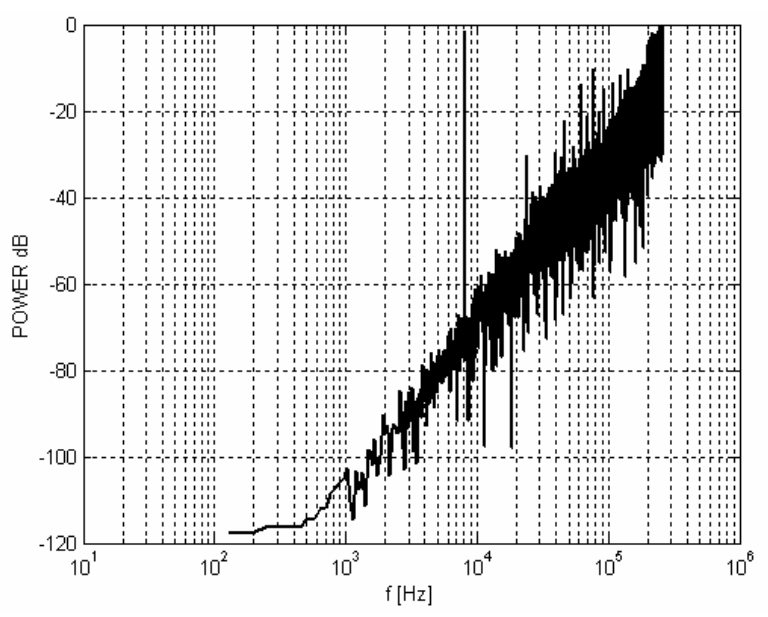

Fig. 8. Output spectrum from transistor level simulation

\section{CONCLUSION}

In this paper, a 2-1 cascaded sigma-delta modulator design has been described. Transistor level simulation results show that the designed circuit fulfills the imposed requirements. Modulator is designed using Cadence Design System and AMI Semiconductors CMOS $0.35 \mu \mathrm{m}$ (C0352P5M-AS) technology. Currently chip is in fabrication phase.

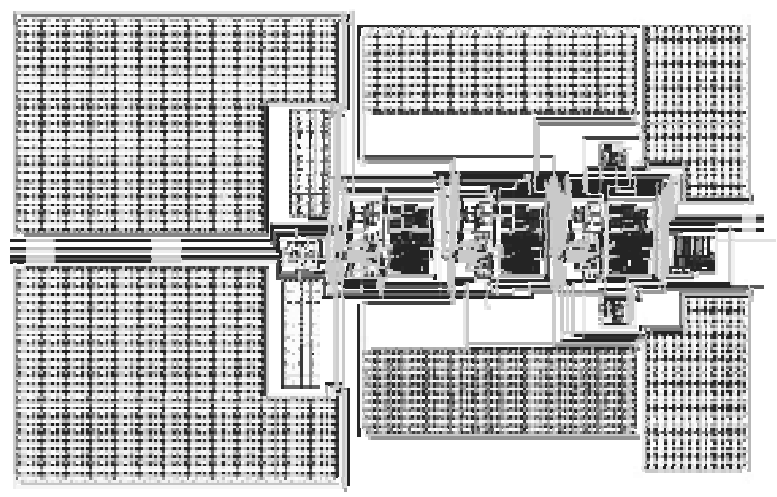

Fig. 9 Layout of the modulator

\section{REFERENCES}

[1] J. Candy and G. Temes, "Oversampling methods for A/D and D/A conversion", in Oversampling Delta-Sigma Data Converters. New York: IEEE Press, 1992, pp. 1-29.

[2] Y. Matsuya, K. Uchimura, A. Iwata, T. Kobayashi, M. Ishikava, and T. Yoshitome, "A 16-bit oversampling A-to-D conversion technology using triple-integration noise shaping", IEEE J. Solid-State Circuits, vol. SC-22, pp. 921-929, Dec. 1987.

[3] L.Williams and B. Wolley, "A third-order sigma-delta modulator with extended dynamic range", IEEE J. Solid-State Circuits, vol. 29, pp. 193-202, March 1994.

[4] K. Nagaraj, J. Vlach, T. Viswanathan, and K. Singhal,"Switched-capacitor integrator with reduced sensitivity to amplifier gain", Electron Lett., vol. 22, pp. 1103-1105, Oct. 1986.

[5] K. Lee and R. Meyer, "Low-distortion switched-capacitor filter design technique" IEEE J. Solid-State Circuit, vol. SC20, pp. 1103-1113, Dec. 1985.

[6] Piero Malcovati Simona Brigati, Fabrizio Francesconi, Franco Maloberti, Paolo Cusinato, and Andrea Baschiroto "Behavioral modeling of switched-capacitor sigma-delta modulators" IEEE Trans. Circuits Syst., vol. 50, pp. 352-364, March 2003.

[7] B. Brandt and B. Wolley, "A $50 \mathrm{MHz}$ muti-bit sigma-delta modulator for 12-b 2-MHz A/D conversion" IEEE J. SolidState Circuit, vol. 26, pp. 1746-1756, Dec. 1991.

[8] Cadence 2003 Documentation

[9] SIMULINK and MATLAB Users Guides, The MathWorks, Inc., Natick, MA, 1997. 